\title{
ANALYSIS OF A MODEL IN SOLAR MAGNETOHYDROSTATICS
}

\author{
DAVID EBERLY \\ Division of Mathematics, Computer Science, and Statistics \\ University of Texas \\ San Antonio, Texas 78285 \\ (Received June 7, 1988 and in revised form January 26, 1989)
}

ABSTRACT. 1 ne model for resistive diffusion of a force-free magnetic field in a compressible plasma is analyzed. Such a model has been suggested for describing the behavior of the solar outer atmosphere and that of solar flares. Previous analysis of the model involved assumptions of linear plasma velocity and constant magnetic energy. This paper deals with nonconstant magnetic energy in the cases of linear plasma velocity and planar plasma velocity. Some necessary conditions on the plasma direction vectors are derived. Finally, some aspects of the model assumptions are discussed, including nonnormality of initial data vectors to initial surfaces.

KEY WORDS AND PHRASES. solar magnetohydrostatics, force-free fields 1980 MATHEMATICS SUBJECT CLASSIFICATION. $\quad$ 85A20,85A90

\section{INTRODUCTION}

In this paper we consider the model for resistive diffusion of a force-free magnetic field in a compressible plasma which moves to compensate for changes in the magnetic configuration. Such a model has been suggested by Low [1] for describing the behavior of the solar outer atmosphere and of solar flares.

Let $\vec{B}(t, \vec{x})$ be the magnetic induction with domain $[0, T] \times S \subseteq \mathbb{R} \times \mathbb{R}^{3}$ where $S$ is an unbounded region. Let $\vec{W}(t, \vec{x})$ be the velocity of the plasma. The model for resistive diffusion is given by

$$
\begin{aligned}
& \overrightarrow{B_{t}}=\vec{\nabla} \times(\vec{W} \times \vec{B})+\eta \vec{\nabla}^{2} \vec{B} \\
& \vec{\nabla} \times \vec{B}=\alpha \vec{B} \\
& \vec{\nabla} \cdot \vec{B}=0 \\
& (\vec{\nabla} \times \vec{W}-\alpha \vec{W}) \cdot \vec{B}=0
\end{aligned}
$$

where $\alpha(t, \vec{x}) \neq 0$ is a scalar function and where $\eta>0$ is the constant resistivity of the plasma. In addition, taking the divergence of equation (1.2) and using equation (1.3) yields the relationship

$$
\vec{B} \bullet \vec{\nabla} \alpha=0 .
$$

For a static plasma $(\vec{W}=\overrightarrow{0})$ and an initial force-free magnetic induction $\vec{B}_{0}(\vec{x})=\vec{B}(0, \vec{x})$, ChandrasekharKendall [2] showed that $\vec{B}(t, \vec{x})$ remains force-free for all time if $\alpha$ is constant. Conversely, Jette [3] showed that $\vec{B}$ remains force-free for all time only if $\alpha$ is a constant.

We assume in our development that $\alpha$ is not constant. Consequently, the direction of the magnetic field lines may vary with time at each $\vec{x} \in S$. In order that the magnetic field remains force-free, it is necessary that the plasma velocity vary, say $\vec{W}=\vec{W}(t, \vec{x})$. Equations (1.1) and (1.4) indicate the relationships between $\vec{B}$ and $\vec{W}$ for such a setting.

Low [1] considered equations (1.1)-(1.5) for a special case $\vec{B} \bullet \vec{k}=0, \vec{W}=\omega \vec{k}$, and $\vec{\nabla} \alpha=\alpha_{x} \vec{k}$. He also assumed that the magnetic energy $\frac{1}{2}\|\vec{B}\|^{2}$ is constant. We consider a more general setting in this paper.

2. DERIVATION OF THE MODEL

We briefly review the derivation of the model from Maxwell's equations. A more thorough development can be found in Priest [4]. A continuous single-fluid plasma which is in thermodynamic equilibrium with distribution functions can be modeled by Maxwell's equations: 


$$
\begin{aligned}
& \vec{\nabla} \times \vec{B}=\mu \vec{J}+\frac{1}{c^{2}} \vec{E}_{t} \\
& \vec{\nabla} \cdot \vec{B}=0 \\
& \vec{\nabla} \times \vec{E}=-\vec{B}_{t} \\
& \vec{\nabla} \cdot \vec{E}=\varrho / \varepsilon
\end{aligned}
$$

where $\vec{E}$ is the electric field, $\vec{J}$ is current density, $\mu$ is magnetic permeability, $\epsilon$ is permittivity, and $\varrho$ is the charge density.

Define $E_{0}=|\vec{E}|, B_{0}=|\vec{B}|$, and $W_{0}=|\vec{W}|$. Two basic assumptions are usually made in magnetohydrodynamics:

$$
W_{0} \ll c \text { and } E_{0} \doteq W_{0} B_{0},
$$

so that for typical length $\ell_{0}$ and typical time $t_{0}$,

$$
\frac{E_{0}}{c^{2} t_{0}} \doteq \frac{W_{0} B_{0}}{c^{2} t_{0}}=\frac{W_{0}^{2} B_{0}}{c^{2} \ell_{0}}=\frac{W_{0}^{2}}{c^{2}} \frac{B_{0}}{\ell_{0}} .
$$

Comparing the magnitudes of terms in equation (2.1) and using the above approximation, the displacement current term is negligible: $\left|c^{-2} \vec{E}_{t}\right| \ll 1$. As a mathematical approximation, assume that $\vec{E}_{t}=\overrightarrow{0}$.

Also, a solar plasma is essentially electrically neutral; that is, if the number density of positive ions is $n_{+}$and the number density of negative ions is $n_{-}$, then the charge density is small: $\varrho=\left(n_{+}-n_{-}\right) e \ll 1$. With these assumptions, equations (2.1) and (2.4) become $\vec{\nabla} \times \vec{B}=\mu \vec{J}$ and $\vec{\nabla} \bullet \vec{E}=0$.

Since the plasma is moving with non-relativistic speed, it is subject to an electric field $\vec{W} \times \vec{B}$ in addition to the electric field $\vec{E}$. In the frame of reference moving with the plasma, Ohm's law is given by $\vec{J}=\sigma(\vec{E}+\vec{W} \times \vec{B})$ where $\sigma$ is electric conductivity.

In active regions, if the magnetic field is small, then the Lorents force is negligible and the magnetic field is approximately force-free. As a mathematical approximation: $\vec{J} \times \vec{B}=0$ or $\mu \vec{J}=\alpha \vec{B}$ for some scalar function $\alpha(t, \vec{x})$.

Ohm's law and the force-free assumption imply $\vec{E}=\eta \alpha \vec{B}-\vec{W} \times \vec{B}$ where $\eta=(\sigma \mu)^{-1}>0$. Combining the above derivations with (2.1)-(2.4) and using $\vec{\nabla} \times(\alpha \vec{B})=-\vec{\nabla}^{2} \vec{B}$ yield the model of resistive diffusion (1.1)- (1.4).

\section{THE MODEL FOR AN IRROTATIONAL PLASMA}

In this section we consider an irrotational plasma, so the velocity $\vec{W}$ satisfies the condition $\vec{\nabla} \times \vec{W}=\overrightarrow{0}$. Consequently, equation (1.4) implies $\vec{B} \bullet \vec{W}=0$. It can be shown that

$$
\vec{\nabla} \times(\vec{W} \times \vec{B})=(\vec{B} \cdot \vec{\nabla}) \vec{W}-(\vec{\nabla} \cdot \vec{W}) \vec{B}-(\vec{W} \cdot \vec{\nabla}) \vec{B}
$$

and

$$
\vec{\nabla}(\vec{B} \bullet \vec{W})=\alpha \vec{W} \times \vec{B}+(\vec{B} \bullet \vec{\nabla}) \vec{W}+(\vec{W} \bullet \vec{\nabla}) \vec{B}
$$

Solving for $(\vec{B} \bullet \vec{\nabla}) \vec{W}$, substituting in equation (1.1) for $\vec{B}$, and grouping terms yields

$$
\vec{B}_{t}+2(\vec{W} \cdot \vec{\nabla}) \vec{B}+\left(\eta \alpha^{2}+\vec{\nabla} \cdot \vec{W}\right) \vec{B}+(\eta \vec{\nabla} \alpha+\alpha \vec{W}) \times \vec{B}=\overrightarrow{0} .
$$

Define the magnetic energy to be $\varepsilon=\frac{1}{2} \vec{B} \bullet \vec{B}$; then dotting the above equation with $\vec{B}$ yields

$$
\varepsilon_{t}+2(\vec{W} \bullet \vec{\nabla}) \varepsilon+2\left(\eta \alpha^{2}+\vec{\nabla} \bullet \vec{W}\right) \mathcal{E}=0 .
$$

Make the change of dependent variable: $\vec{B}=\sqrt{2 \varepsilon} \vec{C}$; then $\vec{C} \bullet \vec{C}=1$ and

$$
\begin{aligned}
& \vec{C}_{t}+2(\vec{W} \bullet \vec{\nabla}) \vec{C}+(\eta \vec{\nabla} \alpha+\alpha \vec{W}) \times \vec{C}=\overrightarrow{0}, \\
& \vec{\nabla} \times \vec{C}-\alpha \vec{C}+\frac{1}{2 \varepsilon} \vec{\nabla} \varepsilon \times \vec{C}=\overrightarrow{0}, \\
& \vec{\nabla} \bullet \vec{C}+\frac{1}{2 \varepsilon} \vec{\nabla} \varepsilon \cdot \vec{C}=0, \\
& \vec{C} \bullet \vec{\nabla} \alpha=0, \quad \vec{C} \bullet \vec{W}=0 .
\end{aligned}
$$


Solutions to (3.1)-(3.4) will be constructed using the right-handed orthonormal system of differentiable vectors $\vec{d}(t, \vec{x}), \vec{v}(t, \vec{x})$, and $\vec{p}(t, \vec{x})=\vec{d} \times \vec{v}$. For notational purposes, the directional derivative $\vec{q} \bullet \vec{\nabla} f$ of a scalar function $f$ will be denoted by $f_{q}$ (where the vector cap on $\vec{q}$ is omitted). Similarly, the directional derivative $(\vec{q} \bullet \vec{\nabla}) \vec{f}$ of a vector function $\vec{f}$ will be denoted $\vec{f}_{q}$.

Choose plasma velocity $\vec{W}=\omega \vec{d}$ where $|\omega| \neq 0$ is the speed of the plasma. Let $\vec{C}=(\cos \psi) \vec{p}+(\sin \psi) \vec{v}$ where $\psi=\psi(t, \vec{x})$. Clearly $\vec{C}$ is a unit vector and the orthogonality conditions in (3.4) are satisfied. Substitute $\vec{C}$ in (3.2) and $\operatorname{dot}$ with $\vec{d}, \vec{v}$, and $\vec{p}$, respectively, to obtain

$$
\begin{aligned}
& (\cos \psi) \psi_{p}+(\sin \psi) \psi_{v}=(\cos \psi)\left(\vec{d} \bullet \vec{\nabla} \times \vec{p}+\frac{\varepsilon_{v}}{2 \varepsilon}\right)+(\sin \psi)\left(\vec{d} \bullet \vec{\nabla} \times \vec{v}-\frac{\varepsilon_{a}}{2 \varepsilon}\right), \\
& -(\sin \psi) \psi_{d}=(\cos \psi)\left(\vec{v} \bullet \vec{\nabla} \times \vec{p}-\frac{\varepsilon_{d}}{2 \varepsilon}\right)+(\sin \psi)(\vec{v} \bullet \vec{\nabla} \times \vec{v}-\alpha), \text { and } \\
& (\cos \psi) \psi_{d}=-(\cos \psi)(\vec{p} \bullet \vec{\nabla} \times \vec{p}-\alpha)-(\sin \psi)\left(\vec{p} \bullet \vec{\nabla} \times \vec{v}+\frac{\varepsilon_{d}}{2 \varepsilon}\right)
\end{aligned}
$$

Substitute $\vec{C}$ in (3.3) to obtain

$$
-(\sin \psi) \psi_{p}+(\cos \psi) \psi_{v}=-(\cos \psi)\left(\vec{\nabla} \cdot \vec{p}+\frac{\mathcal{E}_{p}}{2 \mathcal{E}}\right)-(\sin \psi)\left(\vec{\nabla} \bullet \vec{v}+\frac{\mathcal{E}_{v}}{2 \mathcal{E}}\right)
$$

In solving the above equations, define $\vec{a}=[\cos \psi \sin \psi]^{T}$ and

$$
M=\left[\begin{array}{cc}
\vec{v} \bullet \vec{\nabla} \times \vec{p} & \vec{v} \bullet \vec{\nabla} \times \vec{v} \\
-\vec{p} \bullet \vec{\nabla} \times \vec{p} & -\vec{p} \bullet \vec{\nabla} \times \vec{v}
\end{array}\right], \quad N=\left[\begin{array}{cc}
\vec{d} \bullet \vec{\nabla} \times \vec{p} & \vec{d} \bullet \vec{\nabla} \times \vec{v} \\
\vec{\nabla} \bullet \vec{p} & \vec{\nabla} \bullet \vec{v}
\end{array}\right], \quad R=\left[\begin{array}{cc}
0 & -1 \\
1 & 0
\end{array}\right] ;
$$

then the solutions $\psi_{d}, \psi_{v}$, and $\psi_{p}$ are given by $\psi_{d}=\alpha-\vec{a}^{T} R M \vec{a}, \psi_{v}=-\vec{a}^{T} R N \vec{a}+\frac{\varepsilon_{v}}{2 \varepsilon}$, and $\psi_{p}=\vec{a}^{T} N \vec{a}-\frac{\varepsilon_{v}}{2 \varepsilon}$ with a compatability condition $\frac{\varepsilon_{1}}{\varepsilon}=\vec{a}^{T} M \vec{a}$. Substitute $\vec{C}$ into (3.1) and dot with $\vec{d}$ to obtain

$$
(\cos \psi)\left[\vec{d} \bullet\left(\vec{p}_{t}+2 \omega \vec{p}_{d}\right)+\eta \alpha_{v}\right]+(\sin \psi)\left[\vec{d} \bullet\left(\vec{v}_{t}+2 \omega \vec{v}_{d}\right)-\eta \alpha_{p}\right]=0
$$

Dotting with $\vec{v}$ or $\vec{p}$ produces $\psi_{t}+2 \omega \psi_{d}=\vec{p} \bullet\left(\vec{v}_{t}+2 \omega \vec{v}_{d}\right)+\eta \alpha_{d}+\alpha \omega$. Substituting $\vec{W}=\omega \vec{d}$ in $\vec{\nabla} \times \vec{W}=\overrightarrow{0}$ and dotting with $\vec{d}, \vec{v}$, and $\vec{p}$ yields $\vec{d} \bullet \vec{\nabla} \times \vec{d}=0, \omega_{p}=-\omega(\vec{v} \bullet \vec{\nabla} \times \vec{d}), \omega_{v}=\omega(\vec{p} \bullet \vec{\nabla} \times \vec{d})$, respectively. Finally, substituting $\vec{C}$ in (3.4) yields

$$
(\cos \psi) \alpha_{p}+(\sin \psi) \alpha_{v}=0
$$

Define the matrix

$$
=-\left[\begin{array}{cc}
-\vec{d} \bullet \vec{v}_{t} & \vec{d} \bullet \vec{p}_{t} \\
2 \omega \vec{d} \bullet \vec{p}_{d} & 2 \omega \vec{d} \bullet \vec{v}_{d}
\end{array}\right]
$$

One can solve equations (3.5) and (3.6) for $\alpha_{p}$ and $\alpha_{v}$ in terms of $S$. The general model for an irrotational plasma can be written as

$$
\begin{aligned}
& \psi_{d}=\alpha-\vec{a}^{T} R M \vec{a}, \quad \psi_{v}=-\vec{a}^{T} R N \vec{a}+\frac{\varepsilon_{v}}{2 \mathcal{E}}, \quad \psi_{p}=\vec{a}^{T} N \vec{a}-\frac{\varepsilon_{p}}{2 \mathcal{E}}, \\
& \psi_{t}+2 \omega \psi_{d}=\vec{p} \bullet\left(\vec{v}_{t}+2 \omega \vec{v}_{d}\right)+\eta \alpha_{d}+\alpha \omega, \\
& \frac{\varepsilon_{d}}{\varepsilon}=\vec{a}^{T} M \vec{a}, \quad \varepsilon_{t}+2 \omega \varepsilon_{d}+2\left(\eta \alpha^{2}+\omega \vec{\nabla} \bullet \vec{d}+\omega_{d}\right) \mathcal{E}=0, \\
& \alpha_{p}=\frac{1}{\eta}\left[\vec{a}^{T} S \vec{a}+\vec{v}_{t} \bullet \vec{d}\right], \quad \alpha_{v}=\frac{1}{\eta}\left[\vec{a}^{T} R S \vec{a}-\vec{p}_{t} \bullet \vec{d}\right], \\
& \vec{d} \bullet \vec{\nabla} \times \vec{d}=0, \quad \omega_{p}=-\omega \vec{v} \bullet \vec{\nabla} \times \vec{d}, \quad \omega_{v}=\omega \vec{p} \bullet \vec{\nabla} \times \vec{d}
\end{aligned}
$$

Solutions to these equations provide the plasma velocity $\vec{W}=\omega \vec{d}$, the scalar functions $\alpha$, and the magnetic induction $\vec{B}=\sqrt{2 \mathcal{E}}[(\cos \psi) \vec{p}+(\sin \psi) \vec{v}]$.

\section{LINEAR PLASMA MOTION}

Suppose that the vectors $\vec{d}, \vec{v}$, and $\vec{p}$ are constants. Let $d=\vec{d} \bullet \vec{x}, v=\vec{v} \bullet \vec{x}$, and $p=\vec{p} \bullet \vec{x}$. The model for an irrotational plasma (3.7)- (3.10) reduces to

$$
\begin{aligned}
& \psi_{d}=\alpha, \quad \psi_{v}=\frac{\mathcal{E}_{v}}{2 \mathcal{E}}, \quad \psi_{p}=-\frac{\mathcal{E}_{p}}{2 \mathcal{E}}, \quad \psi_{t}=\eta \alpha_{d}-\alpha \omega \\
& \mathcal{E}_{t}+2\left(\eta \alpha^{2}+\omega_{d}\right) \mathcal{E}=0
\end{aligned}
$$


where $\alpha=\alpha(d, t), \omega=\omega(d, t), \psi=\psi(d, v, p, t)$, and $\mathcal{E}=\mathcal{E}(v, p, t)$. Consequently, $\left(\eta \alpha^{2}+\omega_{d}\right)_{v}=\left(\eta \alpha^{2}+\right.$ $\left.\omega_{d}\right)_{p}=0$. Differentiate $(4.2 b)$ with respect to $d$ to obtain $\left(\eta \alpha^{2}+\omega_{d}\right)_{d}=0$. Thus, $\eta \alpha^{2}+\omega_{d}$ is a function of $t$ only. Moreover, one can solve for the energy function:

$$
\mathcal{E}(t, \vec{x})=\frac{1}{2} \exp \left(-2 \int_{0}^{t} \eta \alpha^{2}(s, d)+\omega_{d}(s, d) d s\right)\left\|\vec{B}_{0}(\vec{x})\right\|^{2},
$$

where $\vec{B}_{0}(\vec{x})=\vec{B}(0, \vec{x})$. The condition $\varepsilon_{d}=0$ forces the initial data to satisfy $(\vec{d} \bullet \vec{\nabla})\left(\left\|\vec{B}_{0}\right\|^{2}\right)=0$.

From the equations $(4.1 \mathrm{~b}, \mathrm{c})$, compute $\psi_{v p}$ in two ways and equate to obtain $(\log \mathcal{E})_{v p}=0$. This forces $\varepsilon_{v} / \mathcal{E}=f(v)$ and $\varepsilon_{p} / \mathcal{E}=g(p)$ for some functions $f$ and $g$. In addition, this implies $\left\|\vec{B}_{0}\right\|^{2}=F(v) G(p)$ for some functions $F$ and $G$. The gradient equation for $\psi$ may be written as

$$
\vec{\nabla} \psi=\alpha \vec{d}+f(v) \vec{v}-g(p) \vec{p} .
$$

Take the $t$-derivative of this equation to obtain $\psi_{d t}=\alpha_{t}$. Take the $d$-derivative of equation (4.1d) to obtain $\psi_{d t}=\left(\eta \alpha_{d}-\alpha \omega\right)_{d}$. Thus, one need only solve for $\alpha$ and $\omega$ using the equations

$$
\alpha_{t}=\eta \alpha_{d d}-(\alpha \omega)_{d}, \quad\left(\eta \alpha^{2}+\omega_{d}\right)_{d}=0 .
$$

One can then solve for $\psi$ from its corresponding equations:

$$
\psi(t, d, v, p)=\sin ^{-1}\left(\frac{\vec{v} \bullet \vec{B}_{0}}{\left\|\vec{B}_{0}\right\|}\right)+\int_{0}^{t}\left[\eta \alpha_{d}(s, d)-\alpha(s, d) \omega(s, d)\right] d s .
$$

Note that in the special case where $\mathcal{E}$ is a constant, it is necessary that $\eta \alpha^{2}+\omega_{d}=0, \alpha_{t}=\eta \alpha_{d d}-(\alpha \omega)_{d}$, and $\psi_{d}=\alpha$, just as in the paper by Low [1].

\section{PLANAR PLASMA MOTION}

Suppose that the plasma velocities $\vec{d}(t, \vec{x})$ all lie in the same plane whose normal vector is the constant vector $\vec{v}$. Without lose of generality assume that $\vec{v}=\vec{k}, \vec{d}=(\cos \gamma, \sin \gamma, 0), \vec{p}=(\sin \gamma,-\cos \gamma, 0)$. The model (3.7)-(3.10) becomes

$$
\begin{aligned}
& \psi_{d}=\alpha-\gamma_{p} \sin \psi \cos \psi, \quad \psi_{v}=\gamma_{d} \cos ^{2} \psi+\frac{\mathcal{E}_{v}}{2 \mathcal{E}}, \quad \psi_{p}=\gamma_{d} \sin \psi \cos \psi-\frac{\mathcal{E}_{p}}{2 \mathcal{E}}, \\
& \psi_{t}+2 \omega \psi_{d}=\eta \alpha_{d}+\alpha \omega, \\
& \frac{\varepsilon_{d}}{\mathcal{E}}=\gamma_{p} \cos ^{2} \psi, \quad \varepsilon_{t}+2 \omega \varepsilon_{d}+2\left(\eta \alpha^{2}+\omega_{d}\right) \mathcal{E}=0, \\
& \alpha_{p}=\frac{1}{\eta}\left(\gamma_{t}+2 \omega \gamma_{d}\right) \sin \psi \cos \psi, \quad \alpha_{v}=-\frac{1}{\eta}\left(\gamma_{t}+2 \omega \gamma_{d}\right) \cos ^{2} \psi, \\
& \omega_{p}=-\omega \gamma_{d}
\end{aligned}
$$

where $\gamma=\gamma(d, p, t)$ and $\omega=\omega(d, p, t)$.

THEOREM 1. In order that the vectors $\vec{d}$ be planar, but not linear, it is necessary that $\vec{\nabla} E \neq \overrightarrow{0}$.

PROOF. Suppose that $\vec{\nabla} E=\overrightarrow{0}$. Then equation (5.2a) implies $\gamma_{p} \cos ^{2} \psi=0$. If $\cos \psi=0$, then $0=\psi_{d}=\alpha$, a contradiction. Therefore, $\gamma_{p}=0$ is necessary. The equations $(5.1 \mathrm{a}, \mathrm{b}, \mathrm{c})$ become $\psi_{d}=$ $\alpha, \quad \psi_{v}=\gamma_{d} \cos ^{2} \psi$, and $\psi_{p}=\gamma_{d} \sin \psi \cos \psi$.

From these equations compute $\psi_{v p}=-2 \gamma_{d}^{2} \sin ^{2} \psi \cos ^{2} \psi=\gamma_{d}^{2}\left(\cos ^{2}-\sin ^{2} \psi\right) \cos ^{2} \psi$, which implies $\gamma_{d} \cos ^{2} \psi=0$. Since $\cos \psi \neq 0$, it must be that $\gamma_{d}=0$. Combining this with $\gamma_{v}=\gamma_{p}=0$ implies $\gamma=\gamma(t)$. We now have $\psi_{d}=\alpha$ and $\psi_{v}=\psi_{p}=0$. These imply $\alpha_{v}=\alpha_{p}=0$, and so equations $(5.3 a, b)$ imply $0=\gamma_{t}+2 \omega \gamma_{d}=\gamma_{t}$. That is, $\gamma$ is a constant and the plasma motion is linear.

EXAMPLE 1. We attempt a solution to the planar model where $\vec{d}=(x / r, y / r, 0), x=r \cos \gamma$, and $y=r \sin \gamma$. Assume that $\alpha=\alpha(r), \omega=\omega(r), \mathcal{E}=\mathcal{E}(t, r)=f(t) g(r)$, and $\psi=\psi(r)$. The model equations become $\psi_{r}=\alpha-\frac{1}{r} \sin \psi \cos \psi, 2 \omega \psi_{r}=\eta \alpha_{r}+\alpha \omega, \frac{\varepsilon_{r}}{\varepsilon}=\frac{1}{r} \cos ^{2} \psi$, and $\mathcal{E}_{t}+2 \omega \mathcal{E}_{r}+2\left(\eta \alpha^{2}+\omega_{r}\right) \mathcal{E}=0$. The assumption of separability of $\mathcal{E}$ implies $\frac{1}{2} f^{\prime}(t) / f(t)+\omega g^{\prime}(r) / g(r)+\eta \alpha^{2}+\omega_{r}=0$. Thus, $f(t)=\exp (-2 b t)$ and $g(r)=a \exp \left(\int_{0}^{r} \omega(s)^{-1}\left[b-\eta \alpha^{2}(s)-\omega_{r}(s)\right] d s\right)$ for some constants $a$ and $b$. 
Moreover, the model equations imply $\sin \psi \cos \psi=\frac{r}{2 \omega}\left(\alpha \omega-\eta \alpha_{r}\right)$ and $\cos ^{2} \psi=\frac{r}{\omega}\left(b-\eta \alpha^{2}-\omega_{r}\right)$. Taking the quotient of these two and solving for $\psi$ yields

$$
\psi=\tan ^{-1}\left(\frac{\alpha \omega-\eta \alpha_{r}}{2\left(b-\eta \alpha^{2}-\omega_{r}\right)}\right) .
$$

The functions $\alpha$ and $\omega$ are determined by the trigonometric relations between $\sin \psi$ and $\cos \psi$, by the equation $\psi_{r}=\frac{\eta \alpha_{r}+\alpha \omega}{2 \omega}$, and by the initial data $g(r)=\varepsilon(0, \vec{x})=\frac{1}{2}\left\|\vec{B}_{0}(\vec{x})\right\|^{2}$.

EXAMPLE 2. There is no solution to the irrotational plasma model for motion vectors of the form $\vec{d}=(-y / r, x / r, 0)$ where $y=-r \cos \gamma$ and $x=r \sin \gamma$. The functions $\alpha, \omega, \varepsilon$, and $\psi$ depend on $r, \gamma, z$, and $t$. The model equations become $\psi_{\gamma}=r \alpha, \quad \psi_{z}=\frac{1}{r} \cos ^{2} \psi+\frac{\varepsilon_{z}}{2 \varepsilon}, \psi_{r}=\frac{1}{r} \sin \psi \cos \psi-\frac{\varepsilon_{r}}{2 \varepsilon}, \varepsilon_{\gamma}=0$, $\varepsilon t+2\left(\eta \alpha^{2}+\omega_{\gamma}\right) \varepsilon=0, \alpha_{r}=\frac{2 \omega}{\eta r} \sin \psi \cos \psi, \alpha_{z}=-\frac{2 \omega}{\eta r} \cos ^{2} \psi, \omega_{x}=0$, and $\omega_{r}=-\frac{w}{r}$.

From these equations compute $\psi_{r \gamma}$ in two ways and equate to obtain $\omega(\cos \psi)+\eta \alpha(\sin \psi)=0$. Similarly, compute $\psi_{x \gamma}$ in two ways and equate to obtain $\omega(\cos \psi)-\eta \alpha(\sin \psi)=0$. Since $\cos \psi \neq 0$ and $\sin \psi \neq 0$, it must be that $\omega=0$ and $\alpha=0$, a contradiction to the assumptions of the model.

\section{THE GENERAL PLASMA MODEL}

Without loss of generality, use the right-handed orthonormal system of differentiable vectors $\vec{d}=$ $(\cos \gamma \sin \phi, \sin \gamma \sin \phi, \cos \phi), \vec{v}=(-\cos \gamma \cos \phi,-\sin \gamma \cos \phi, \sin \phi)$, and $\vec{p}=\vec{d} \times \vec{v}=(\sin \gamma,-\cos \gamma, 0)$ where $\gamma=\gamma(t, \vec{x})$ and $\phi=\phi(t, \vec{x})$. The matrices in the plasma model (3.7)-(3.10) are given by

$$
N=\left[\begin{array}{cc}
\gamma_{p} \sin \phi & \phi_{p}-\gamma_{d} \cos \phi \\
\gamma_{v} \sin \phi+\gamma_{d} \cos \phi & \phi_{v}
\end{array}\right], N=\left[\begin{array}{cc}
\gamma_{p} \cos \phi & \gamma_{v} \cos \phi \\
\gamma_{d} \sin \phi-\gamma_{v} \cos \phi & \phi_{d}+\gamma_{p} \cos \phi
\end{array}\right] \text {. }
$$

and

$$
s=\left[\begin{array}{cc}
-\phi_{t} & \gamma_{t} \sin \phi \\
2 \omega \gamma_{d} \sin \phi & 2 \omega \phi_{d}
\end{array}\right]
$$

The general model is extremely complicated in form. However, one can determine necessary conditions on the plasma velocity for existence of solutions from the compatability condition (3.8a), which in this setting becomes

$$
\frac{\vec{d} \cdot \vec{\nabla} \mathcal{E}}{\mathcal{E}}=\vec{a}^{T}\left[\begin{array}{cc}
\gamma_{p} \sin \phi & \phi_{p} \\
\gamma_{v} \sin \phi & \phi_{v}
\end{array}\right] \vec{a}=: \vec{a}^{T} Q \vec{a}
$$

The condition (3.10a), $\vec{d} \bullet \vec{\nabla} \times \vec{d}=\overrightarrow{0}$, implies $\phi_{p}=\gamma_{v} \sin \phi$. Thus, the matrix $Q$ is symmetric. We consider here the two cases: $\varepsilon_{d}=0$ and $\varepsilon_{d} \neq 0$.

THEOREM 2. Suppose that $\varepsilon_{d}=0$. A necessary condition for existence of solutions to the irrotational plasma model is: $\operatorname{det} Q \leq 0$.

PROOF. The compatability condition $\vec{a}^{T} Q \vec{a}=0$ is a quadratic equation which represents a degenerate conic section. Factor $Q=R^{T} \Lambda R$ where $R$ is orthogonal and $\Lambda$ is diagonal, say $\Lambda=\operatorname{diag}\left(\lambda_{1}, \lambda_{2}\right)$. The quadratic equation is then equivalent to $\lambda_{1} \xi_{1}^{2}+\lambda_{2} \xi_{2}^{2}=0$ where $\vec{\xi}=\left[\begin{array}{ll}\xi_{1} & \xi_{2}\end{array}\right]^{T}=R \vec{a}$. If $\operatorname{det} Q>0$, then $\operatorname{sgn}\left(\lambda_{1}\right)=\operatorname{sgn}\left(\lambda_{2}\right) \neq 0$. Consequently, $\vec{\xi}=\overrightarrow{0}$, so $\vec{a}=\overrightarrow{0}$. But this is a contradiction to $\|\vec{a}\|=1$. Thus, it is necessary that $\operatorname{det} Q \leq 0$.

Note that if $\operatorname{det} Q<0$, then the two equations $\vec{a}^{T} Q \vec{a}=0$ and $\|\vec{a}\|=1$ have four solutions (given in terms of $\vec{\xi})$ :

$$
\pm\left(\sqrt{\frac{\lambda_{2}}{\lambda_{2}-\lambda_{1}}}, \sqrt{\frac{\lambda_{1}}{\lambda_{1}-\lambda_{2}}}\right), \pm\left(\sqrt{\frac{\lambda_{2}}{\lambda_{2}-\lambda_{1}}},-\sqrt{\frac{\lambda_{1}}{\lambda_{1}-\lambda_{2}}}\right) .
$$

It may be that these lead to multiple solutions for the magnetic induction $\vec{B}$ and scalar function $\alpha$, for the same plasma velocity $\vec{W}$.

THEOREM 3. Suppose that $\mathcal{E}_{d} \neq 0$. A necessary condition for the existence of solutions to the irrotational plasma model is: $\operatorname{det} Q \leq 0$ or $\operatorname{sgn}(\operatorname{trace}(Q))=\operatorname{sgn}\left(\frac{\varepsilon_{\varepsilon}}{\varepsilon}\right)$.

PROOF. As in Theorem 2, factor $Q=R^{T} \Lambda R$ where $R$ is orthogonal and $\Lambda=\operatorname{diag}\left(\lambda_{1}, \lambda_{2}\right)$. One obtains a quadratic equation $\lambda_{1} \xi_{1}^{2}+\lambda_{2} \xi_{2}^{2}=\varepsilon_{d} / \mathcal{E}$ where $\vec{\xi}=R \vec{a}$. There are no solutions to this equation if $\operatorname{sgn}\left(\lambda_{1}\right)=\operatorname{sgn}\left(\lambda_{2}\right)=-\operatorname{sgn}\left(\frac{\varepsilon_{\alpha}}{\varepsilon}\right)$. This set of equations is equivalent to $\operatorname{det}(Q)>0$ and $\operatorname{sgn}(\operatorname{trace}(Q))=$ 
$-\operatorname{sgn}\left(\frac{\varepsilon_{1}}{\varepsilon}\right)$. Therefore it is necessary for existence of solutions to the plasma model that $\operatorname{det}(Q) \leq 0$ or $\operatorname{sgn}(\operatorname{trace}(Q))=\operatorname{sgn}\left(\frac{\varepsilon_{\alpha}}{\varepsilon}\right)$.

Define $c=\varepsilon_{d} / \varepsilon$. Suppose that $\frac{\lambda_{1}}{c}>0$ and $\frac{\lambda_{2}}{c}>0$. The quadratic equation in Theorem 3 defines an ellipse in $\mathbb{R}^{2}$ with center at the origin and whose axis lengths (measured from the origin) are $\ell_{1}=\sqrt{c / \lambda_{1}}$ and $\ell_{2}=\sqrt{c / \lambda_{2}}$. If the plasma model has solutions, then it is necessary that this ellipse intersect the unit circle. This happens if $\ell_{1} \leq 1 \leq \ell_{2}$ or if $\ell_{1} \geq 1 \geq \ell_{2}$. In either case, there are four solutions to $\vec{a}^{T} Q \vec{a}=c$ and $\|\vec{a}\|=1$.

Similarly, if $\frac{\lambda_{1}}{c}>0$ and $\frac{\lambda_{2}}{c}<0$, then the quadratic equation in Theorem 3 defines a hyperbola in $\mathbb{R}^{2}$ with center at the origin. The distance from the origin to the hyperbola is given by $\ell=\sqrt{c / \lambda_{2}}$. If the plasma model has solutions, then it is necessary that $\ell \leq 1$. In this case, there are also four solutions to $\vec{a}^{T} Q \vec{a}=c$ and $\|\vec{a}\|=1$. Analogous to the setting for Theorem 2, these solutions may produce different magnetic inductions $\vec{B}$ and scalar functions $\alpha$ for the same plasma velocity $\vec{W}$.

\section{OBSERVATIONS}

One basic assumption for the plasma model is that of electrical neutrality given by $\varrho \ll 1$. One can interpret this assumption as $\varrho=0$ or one can treat the problem from the point of view of continuous dependence. In either case, the electric divergence equation cannot be ignored in the development of the model.

For example, Low [1] had attempted a solution of the solar plasma model of the form $\vec{B}(r, t)=B_{1} \hat{r}+$ $B_{2} \hat{\theta}+B_{3} \hat{z}$ where $x=r \cos \theta$ and $y=r \sin \theta$. Consequently, $B_{1}=0, B_{3, r}=-\alpha B_{2},\left(r B_{2}\right)_{r}=\alpha r B_{3}$, $B_{2, t}-\eta\left[\frac{1}{r}\left(r B_{2, r}\right)_{r}-\frac{1}{r^{2}} B_{2}\right]+\left(v_{1} B_{2}\right)_{r}=v_{2} B_{3}-v_{3} B_{2}$, and $B_{3, t}-\eta \frac{1}{r}\left(r B_{3, r}\right)_{r}+\frac{1}{r}\left(r v_{1} B_{3}\right)=0$ where the velocity is given by $\vec{v}(t, \hat{x})=v_{1}(t, r) \hat{r}+z\left(v_{2}(t, r) \hat{\theta}+v_{3}(t, r) \hat{z}\right)$.

A steady solution of these equations is $\vec{B}(r)=a r\left(1+a^{2} r^{2}\right)^{-1} \hat{\theta} \pm\left(1+a^{2} r^{2}\right)^{-1} \hat{z}, \alpha(r)= \pm 2 a\left(1+a^{2} r^{2}\right)^{-1}$, and $\vec{v}(r, z)=2 \eta a\left[-a r\left(1+a^{2} r^{2}\right)^{-1} \hat{r} \mp 2 a^{2} r z\left(1+a^{2} r^{2}\right)^{-1} \hat{\theta}\right]$ where $a$ is an arbitrary constant. It must be pointed out that these functions do not satisfy equation (1.4). More precisely, the condition $\vec{B} \bullet(\vec{\nabla} \times \vec{v}-\alpha \vec{v})=0$ is not satisfied. It can be shown that

$$
\varrho=\vec{\nabla} \cdot \vec{E}=-\vec{B} \bullet(\vec{\nabla} \times \vec{v}-\alpha \vec{v})=\frac{4 a^{2} z\left(1-a^{2} r^{2}\right)}{\left(1+a^{2} r^{2}\right)^{3}} \neq 0
$$

for $a \neq 0$. Moreover, for $|z|$ sufficiently large, $|\varrho|$ is large, a contradiction to the assumption of electric neutrality.

We also provide an answer to a question by Priest [4] on the conjecture of non- normality of initial data $\vec{B}_{0}(\vec{x})$ to any smooth initial surface for the force-free model $\vec{\nabla} \times \vec{u}=\alpha \vec{u}, \vec{\nabla} \bullet \vec{u}=0$. These equations are equivalent to

$$
A \vec{u}_{x}+B \vec{u}_{y}+C \vec{u}_{z}=\alpha D \vec{u}
$$

where $A, B, C, D$ are $4 \times 3$ constant matrices whose entries are 0,1 , or -1 .

Let $\vec{\rho}(s, t)$ be a $C^{2}$-surface in $\mathbb{R}^{3}$ which acts as the initial surface for the force-free model. Define $\vec{\phi}(s, t)=\vec{u}(\vec{\rho}(s, t))$ as the initial data on the surface. Then

$$
\vec{\rho}_{0}=x_{0} \vec{u}_{x}+y_{0} \vec{u}_{y}+z_{0} \vec{u}_{z} \text { and } \vec{\rho}_{t}=x_{t} \vec{u}_{x}+y_{t} \vec{u}_{y}+z_{t} \vec{u}_{x}
$$

Consider the equations (7.1) and (7.2) evaluated on the initial surface. Define the $4 \times 1$ column vector

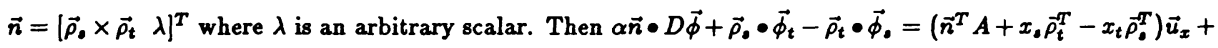
$\left(\vec{n}^{T} B+y_{0} \vec{\rho}_{t}^{T}-y_{t} \vec{\rho}_{0}^{T}\right) \vec{u}_{y}+\left(\vec{n}^{T} C+z_{0} \vec{\rho}_{t}^{T}-z_{t} \vec{\rho}_{0}^{T}\right) \vec{u}_{z}=\lambda \vec{\nabla} \bullet \vec{u}=0$. Therefore,

$$
\alpha\left(\vec{\rho}_{0} \times \vec{\rho}_{t} \bullet \vec{\phi}\right)+\vec{\rho}_{0} \bullet \vec{\phi}_{t}-\vec{\rho}_{t} \bullet \vec{\phi}_{0}=0 .
$$

THEOREM 4. The initial data $\vec{\phi}(s, t) \neq \overrightarrow{0}$ cannot be everyvihere normal on any open subset of the surface $\vec{\rho}(s, t)$. 
PROOF. Suppose that $\vec{\phi}$ is normal everywhere on an open subset of the surface. Then $\vec{\phi}=K(s, t) \vec{\rho}_{0} \times \vec{\rho}_{t}$. Replacing this in equation (7.3) yields $\alpha K\left\|\vec{\rho}_{0} \times \vec{\rho}_{t}\right\|^{2}=0$. But $\alpha \neq 0, \vec{\rho}_{0} \times \vec{\rho}_{t} \neq \overrightarrow{0}$, and $K \neq 0$, a contradiction. Thus, $\vec{\phi}$ is not everywhere normal to the initial surface.

\section{REFERENCES}

1. LOW, B.C., Resistive diffusion of force-free magnetic fields in a passive medium, The Astrophysical Journal 181 (1973), 209-226.

2. CHANDRASEKHAR, S. and KENDALL, P.C., On force-free magnetic fields, The Astrophysical Journal 126 (1957), 457-460.

3. JETTE, A.D., Force-free magnetic fields in resistive magnetohydrostatics, J. Math. Anal. Appl. 29 (1970), 109+.

4. PRIEST, E.R., “Solar Magnetohydrodynamics” (Geophyics and Astrophysics Monographs), D.Reidel Publishing Co., Dordrecht 1984. 


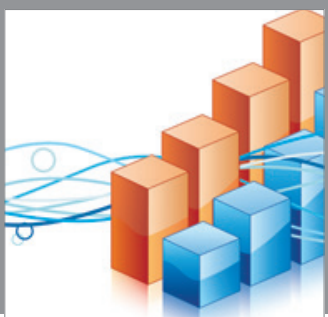

Advances in

Operations Research

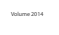

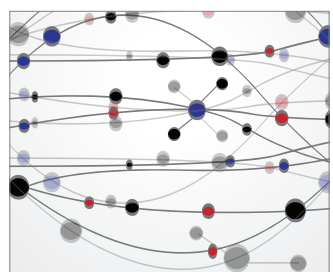

\section{The Scientific} World Journal
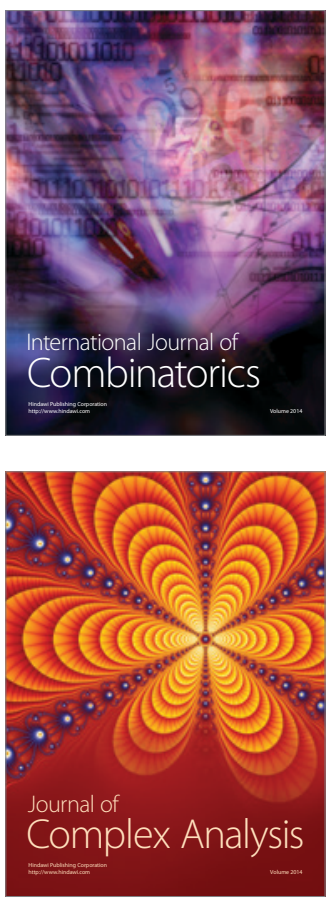

International Journal of

Mathematics and

Mathematical

Sciences
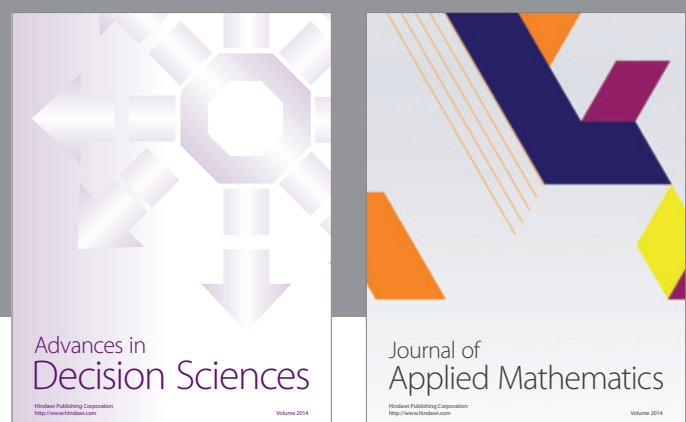

Journal of

Applied Mathematics
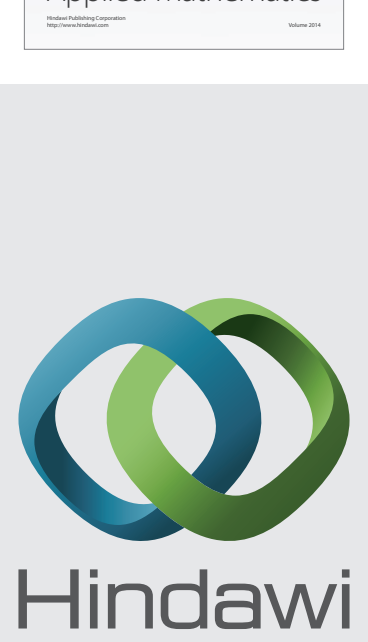

Submit your manuscripts at http://www.hindawi.com
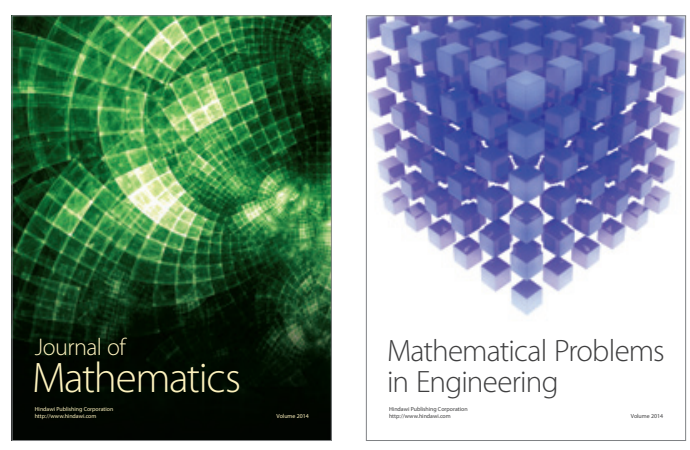

Mathematical Problems in Engineering
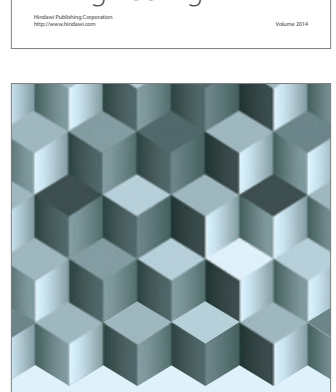

Journal of

Function Spaces
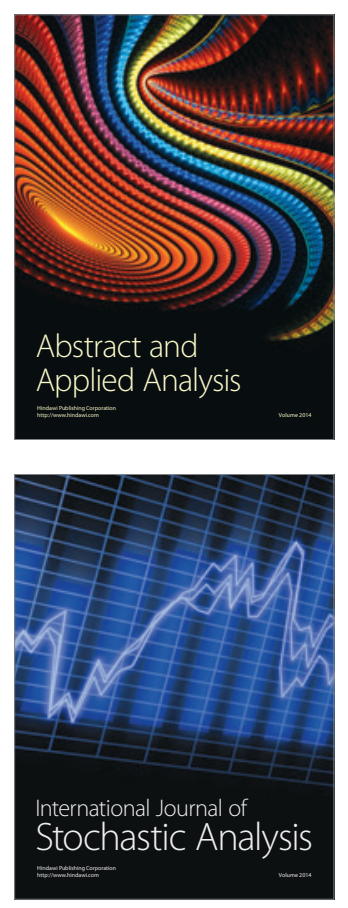

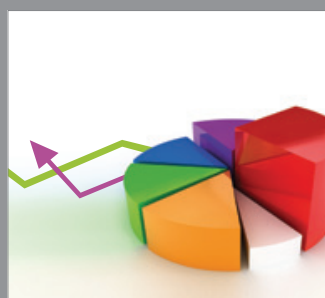

ournal of

Probability and Statistics

Promensencen
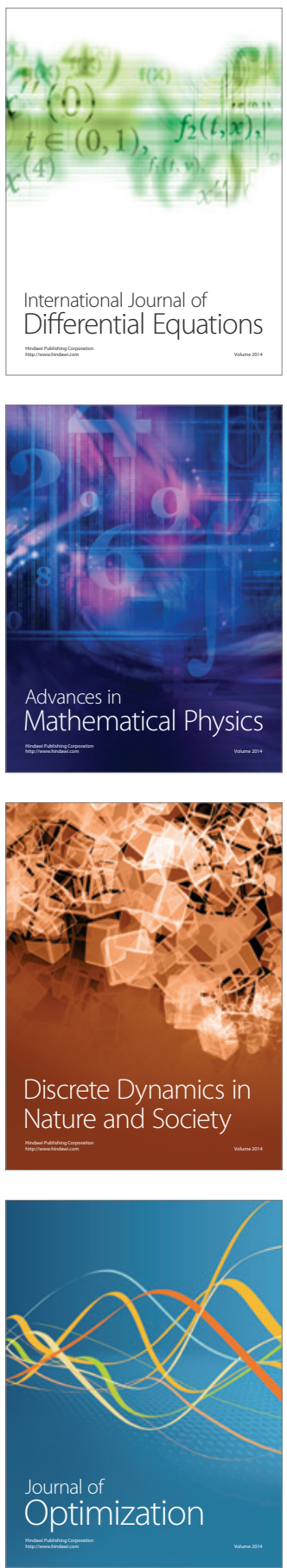